\title{
On the Integral Representation of States on a $C^{*}$-Algebra
}

\author{
Dang-Ngoc-Nghiem* \\ Université Paris VI. Laboratoire de Probabilités, Paris, France
}

Received January 30, 1974; in revised form July 6, 1974

\begin{abstract}
The purpose of this paper is to give some complements to the various extremal decompositions of states on a $C^{*}$-dynamical system i.e. a pair $(A, G)$ where $A$ is a $C *$-algebra and $G$ is a group acting on $A$ by $*$-automorphisms. We shall see for instance that the method of decomposition associated with a maximal abelian $W^{*}$-algebra does not give all the extremal measures in the general case. We also give the explicit form of the greatest lower bound of all the extremal measures and a certain form of continuity of the decomposition. Finally we characterize various systems in the literature ( $G$-abelian algebras, large systems and quasi-large systems) in terms of the equivalence of different notions of ergodicity.
\end{abstract}

\section{Introduction and Notations}

Let $A$ be a $C^{*}$-algebra with identity, $G$ a group and $\tau$ a representation of $G$ in the $*$-automorphism-group $\operatorname{Aut}^{*}(A)$ of $A$; in a number of recent articles, the invariant states of $A$ and their integral representation have been intensively studied under certain conditions ( $G$-abelian algebras, asymptotically abelian systems, large systems, etc... (cf. [7, 8, 10,12,13]) and more recently Guichardet and Kastler have studied the integral representation of quasi-invariant states (cf. [8]). These systems have many applications in Physics, particularly in Statistical Mechanics (cf. $[8,12]$ ).

The purpose of this paper is to give some complements to the various extremal decompositions in the general case and to find necessary and sufficient conditions for the uniqueness of the decomposition; we shall see, for instance, that the method of decomposition associated with a maximal abelian $W^{*}$-algebra does not give all the extremal measures in the general case; we also give the explicit form of the greatest lower bound of all extremal measures and a certain form of continuity of the decompositions.

Finally we characterize various systems cited above in terms of their ergodic states, we give in particular the converse of a result of Ruelle on $G$-abelian algebras and the converse of a result of Haag, Kastler, Michel and Nagel on "quasi-large" systems.

The author would like to thank Professors A. Guichardet and D. Ruelle for their advice and encouragement and Professor G. W. Mackey for the hospitality at Harvard University where part of this work was done.

Notations. Throughout this note, we use the following notations: $A$ is a $C^{*}$-algebra with identity $1, G$ is a group, $\tau$ is a representation of $G$ into $\operatorname{Aut}^{*}(A)$,

* Equipe de Recherche $n^{\circ} 1$ "Processus stochastiques et Applications" dépendant de la section $n^{\circ} 1$ "Mathématiques Informatique" associée au C.N.R.S. (France). 
$E$ is the convex compact set of states on $A, I$ is the convex compact set of invariant states (for $\tau$ ) on $A$; if $a \in A$, the function $\hat{a}$ on $E$ is defined by

$$
\hat{a}(s)=s(a), \quad s \in E .
$$

For a fixed invariant state $s \in I$, the canonical cyclic representation associated with $s$ is $\left(\mathfrak{H}_{s}, \pi_{s}, \xi_{s}\right)$ or simply $(\mathfrak{H}, \pi, \xi)$ if there is no confusion; the canonical unitarity representation $U^{s}$ (or simply $U$ ) satisfies

$$
\pi_{s}\left(\tau_{g} a\right)=U_{g}^{s} \pi_{s}(a) U_{g}^{s^{-1}}, \forall U_{g}^{s} \xi_{s}=\xi_{s}, \quad \forall a \in A, \quad g \in G .
$$

We denote $U_{G}^{s}=\left\{U_{g}^{s}\right\}_{g \in G}$ and $\mathscr{R}_{s}=\left\{\pi_{s}(A) \cup U_{G}^{s}\right\}^{\prime \prime}$, we remark that $\mathscr{R}_{s}^{\prime}$ is the set of elements of $\pi_{s}(A)^{\prime}$ invariant under the action of the mappings $T \rightarrow U_{g} T U_{g}^{-1}$, $g \in G$.

If $B$ is an abelian $W^{*}$-subalgebra of $\pi_{s}(A)^{\prime}, v_{B}^{s}$ (or $v_{B}$ or $v$ if no confusion is possible) is the $B$-measure of $s$ (cf. [14] 3-1-2).

Let $\Omega(s)$ be the set of all probability Radon measure $\mu$ on $E$ satisfying

$$
s(a)=\int_{E} \hat{a}(\psi) d \mu(\psi), \quad \forall a \in A .
$$

Let $\Omega^{I}(s)$ be the set of measures of $\Omega(s)$ with support in $I$ and let $\prec$ be the Choquet-Bishop-de Leeuw order on $\Omega(s)$ and $\Omega^{I}(s)$ (cf. [1]) $\delta_{s}$ denotes the Dirac measure at the point $s$.

\section{Integral Representation of Invariant States}

We summarize some classical results that will be useful in this Lemma:

Lemma 0. a) $\delta_{s} \prec \mu$ for every measure $\mu$ with barycenter $s$.

b) Cartier-Fell-Meyer theorem (cf. [2]): the following conditions are equivalent:

(i). $v \prec \mu$.

(ii). If $v=\sum_{i=1}^{n} v_{i}$ with $v_{i}$ positive measures, then there exist measures $\mu_{i} \geqq 0$, $i=1, \ldots, n$, such that $\mu=\sum_{i=1}^{n} \mu_{i}$ and barycenter $\left(\mu_{i}\right)=\operatorname{barycenter}\left(v_{i}\right)$.

c) Let $\mu=\sum_{j=1}^{n} \alpha_{j} \delta_{s_{j}} \alpha_{j}>0, \sum_{j=1}^{n} \alpha_{j}=1$, barycenter $(\mu)=s$, as $\alpha_{j} s_{j} \leqq s$, there exist $b_{j}$ (unique) in $\pi_{s}(A)_{+}^{\prime}$ such that $\sum_{j=1}^{n} b_{j}=1$ and

$$
\alpha_{j} s_{j}(a)=<\pi_{s}(a) b_{j} \xi_{s}, \quad \xi_{s}>, \quad \forall a \in A .
$$

d) (cf. [13] Corollar 1.4) Let $B$ be an abelian $W^{*}$-algebra of $\pi_{s}(A)^{\prime}$, let $\left\{b_{j}\right\}$ be a finite set of positive elements of $B$ such that $\sum_{j} b_{j}=1$, we define $\alpha_{j} \geqq 0$ and $s_{j} \in E$ by

$$
\alpha_{j}=\left\langle\xi_{s}, b_{j} \xi_{s}\right\rangle, \quad \alpha_{j} s_{j}(a)=\left\langle\pi_{s}(a) \xi_{s}, \xi_{s}\right\rangle, \quad \forall a \in A .
$$

The measure $\mu_{\left\{b_{j}\right\}}=\sum_{j} \alpha_{j} \delta_{s_{j}}$ is called a discrete B-measure. The discrete Bmeasures form a directed filter converging vaguely to $v_{B}$.

e) (cf. [13] Corollar 1.5) Let $B$ and $\tilde{B}$ be two abelian $W^{*}$-algebras of $\pi_{s}(A)^{\prime}$. Then $(\tilde{B} \subset B) \Leftrightarrow\left(v_{\tilde{B}} \prec v_{B}\right)$.

f) Let $B$ be an abelian $W^{*}$-algebra of $\pi_{s}(A)^{\prime}, s \in I$. Then

$$
\left(v_{B} \in \Omega^{I}(s)\right) \Leftrightarrow\left(\operatorname{Supp}\left(v_{B}\right) \subset I\right) \Leftrightarrow\left(B \subset \mathscr{R}_{s}^{\prime}\right) .
$$




\section{Extremal Decomposition of Invariant States - Uniqueness - Simplicial Systems}

Lemma 1. Let $s$ be a state on $A$; if $\pi_{s}(A)^{\prime}$ is abelian, then the $\pi_{s}(A)^{\prime}$-measure $v$ of $s$ is the unique extremal measure on $E$ with barycenter $s$.

Proof. We observe by the Lemma 0c) that each finite discrete measure of $\Omega(s)$ is a discrete $\pi_{s}(A)^{\prime}$-measure. The lemma follows immediately.

Theorem 1. (Extremal decomposition of invariant states). Let $B$ be an abelian $W^{*}$-subalgebra of $\mathscr{R}_{s}^{\prime}, s \in I$, let $v_{B}^{s}$ be the B-measure of $s$. Then

a) If $v_{B}^{s}$ is maximal in $\Omega^{I}(s)$ with respect to Choquet-Bishop-de Leeuw order then $B$ is maximal abelian in $\mathscr{R}_{s}^{\prime}$.

b) If $B$ is maximal abelian in $\mathscr{R}_{s}^{\prime}$, then for any Baire subset $\Delta$ of $I$ such that $\Delta \cap \mathscr{E}(I)=\emptyset(\mathscr{E}(I)$ is the set of extremal points of $I) v_{B}^{s}(\Delta)=0$; in particular if $A$ is separable, the measure $v_{B}^{s}$ is maximal for the order $\prec$.

Proof. The part a) of the theorem is immediate.

Now let $B$ be a maximal abelian $W^{*}$-subalgebra of $\mathscr{R}_{s}^{\prime} ; e$ is the orthogonal projection on $\left[\overline{B \xi_{s}}\right], D$ is the $C^{*}$-algebra generated by $\left(\pi_{s}(A) \cup U_{G} \cup B\right), \tilde{E}$ is the set of states on $D$ and $\tilde{s}$ is the state defined by

$$
\tilde{s}(d)=<d \xi_{s}, \quad \xi_{s}>, \quad d \in D .
$$

We have

$$
\begin{aligned}
& D^{\prime}=\left(\pi_{s}(A) \cup U_{G} \cup B\right)^{\prime}, \\
& D^{\prime}=\mathscr{R}_{s}^{\prime} \cap B^{\prime}, \\
& D^{\prime}=B .
\end{aligned}
$$

Let $\tilde{v}_{B}$ be the $B$-measure of $\tilde{s}$ on $\tilde{E}$.

1) By the Lemma 1, $\tilde{v}_{B}$ is the only extremal measure on $\tilde{E}$ with barycenter $\tilde{s}$.

2) The group $G$ acts on $D$ in canonical way: $d \rightarrow U_{g} d U_{g}^{-1}, d \in D$. Let $\tilde{I}$ denote the set of invariant states on $D$ and let $\tilde{J}$ be the convex compact subset of $\tilde{I}$ defined by $\tilde{J}=\left\{\tilde{\phi} \in \tilde{I} / \tilde{\phi}\left(U_{g}\right)=1, \forall g \in G\right\}$; we can easily see that if $\tilde{\mu}$ is a discrete measure on $\tilde{E}$ such that $\tilde{\mu} \prec \tilde{v}_{B}$, then $\operatorname{supp}(\tilde{\mu}) \subset \tilde{J}$; using the vague limit and the weak of $\tilde{J}$, we obtain $\operatorname{supp}\left(\tilde{v}_{B}\right) \subset \tilde{J}$.

3) Consider the $C^{*}$-algebra $\pi_{s}(A)$, let $E^{\prime}$ be its state space, let $I^{\prime}$ be the set of invariant states of $E^{\prime}$, and for $\tilde{\phi} \in \tilde{E}$, let $\left.\tilde{\phi}\right|_{\pi_{s}(A)}$ be the restriction of $\tilde{\phi}$ to $\pi_{s}(A)$. Put $\gamma(\tilde{\phi})=\left.\tilde{\phi}\right|_{\pi_{s}(A)}, \gamma$ is a continuous mapping of $\tilde{E}$ on $E^{\prime}$. Let $v^{\prime}=\gamma\left(\tilde{v}_{B}\right)$, it is clear that $v^{\prime}$ is the $B$-measure of $\gamma(\tilde{s})$ on $E^{\prime}$ and $\operatorname{supp}\left(v^{\prime}\right) \subset I^{\prime}$.

We can identify $E^{\prime}$ to a compact subset of $E$ and $I^{\prime}$ to a compact subset of $I$; so we can write $v^{\prime}=v_{B}^{s}=\gamma\left(\tilde{v}_{B}\right)$.

To finish the proof we shall show that if $\Delta$ is a subset of $I$ such that $\Delta \cap \mathscr{E}(I)=\emptyset$ then $\gamma^{-1}(\Delta) \cap \tilde{J} \cap \mathscr{E}(\tilde{E})=\Phi\left(\right.$ for $\tilde{v}_{B}$ is an extremal measure on $\tilde{E}$ and $\left.\operatorname{supp}\left(\tilde{v}_{B}\right) \subset \tilde{J}\right)$.

Suppose that $\tilde{\phi} \in \gamma^{-1}(\Delta) \cap \tilde{J} \cap \mathscr{E}(\tilde{E})$ and let $\phi=\gamma(\tilde{\phi})$; consider the cyclic representation of $D$ associated with $\tilde{\phi}:(\tilde{\mathfrak{H}}, \tilde{\pi}, \tilde{\xi})$ and let $\tilde{U}_{g}=\tilde{\pi}\left(U_{g}\right), g \in G$. We have

$$
1=\tilde{\phi}\left(U_{g}\right)=\left\langle\tilde{\pi}\left(U_{g}\right) \tilde{\xi}, \tilde{\xi}\right\rangle=\left\langle\tilde{U}_{g} \tilde{\xi}, \tilde{\xi}\right\rangle=\|\tilde{\xi}\|^{2}
$$

and

$$
\left\|\tilde{U}_{g} \tilde{\xi}\right\|=\|\tilde{\xi}\|
$$


Therefore

$$
\tilde{U}_{g} \tilde{\xi}=\tilde{\xi}, \quad \forall g \in G .
$$

The set of vectors $\tilde{U}_{G} \tilde{\xi}, \tilde{\pi}(A) \tilde{\xi}, \tilde{\pi}(B) \tilde{\xi}$ generates $\tilde{\mathfrak{H}}$, as $\tilde{U}_{g} \tilde{\xi}=\tilde{\xi}$ and $\pi(b)$ is a scalar operator, for $b \in B$ (since $B$ is in the center of $D$ and $\tilde{\pi}$ is irreducible), we have

$$
\left(\overline{\tilde{\pi} \cdot \pi_{s}(A) \xi}\right)=\tilde{\mathfrak{H}}
$$

and the representation $\tilde{\pi} \cdot \pi_{\mathfrak{\xi}}$ of $A$ in $\tilde{\mathfrak{H}}$ is the canonical cyclic representation associated with the state $\phi, \tilde{\xi}$ is the cyclic vector, and we have

$$
\tilde{\pi} \cdot \pi_{s}\left(\tau_{g} a\right)=\tilde{U}_{g} \tilde{\pi} \cdot \pi_{s}(a) \tilde{U}_{g}^{-1}, \quad \forall a \in A, \quad \forall g \in G .
$$

Since $\tilde{\phi}$ is a pure state on $D$, we have

$$
\left(\tilde{\pi}\left(\pi_{s}(A)\right) \cup \tilde{U}_{g}\right)^{\prime}=\tilde{\pi}(D)^{\prime}=C .1_{\mathfrak{H}} .
$$

This relation proves that $\phi$ is an extremal state of $I$ i.e. $\phi \in \Delta \cap \mathscr{E}(I)$ that contradicts the hypotheses. q.e.d.

Definition. A system $(A, G, \tau)$ such that the set of invariant states is a simplex is called a simplicial system.

We shall see (cf. [4]) that the notion of simplicial system coincides with that of $G$-abelian algebra introduced by Lanford and Ruelle (cf. $[12,13]$ ).

\section{Examples of Extremal Invariant Measures Not Associated with an Abelian W*-Subalgebra}

The following proposition shows that we cannot obtain all the extremal invariant measures by taking measures associated with abelian $W^{*}$-algebras in the general case.

Proposition 1. If an invariant state $s \in I$ is such that $\mathscr{R}_{s}^{\prime}$ is not abelian then there exist extremal measures that are not associated with an abelian $W^{*}$-subalgebra of $\mathscr{R}_{s}^{\prime}$.

Proof. Let $b_{1}, b_{2} \in \mathscr{R}_{s+}^{\prime}, b_{1} b_{2} \neq b_{2} b_{1}$ and $b_{1}+b_{2} \leqq 1$, and let $b_{3}=1-b_{1}-b_{2}$, $\alpha_{j}=\left\langle b_{j} \xi_{s}, \xi_{s}\right\rangle$ and

$$
s_{j}(a)=\frac{1}{\alpha_{j}}<\pi_{s}(a) b_{j} \xi_{s}, \quad \xi_{s}>, \quad a \in A, \quad j=1,2,3 .
$$

the discrete measure $\mu$ on $E$ defined by

$$
\mu=\sum_{j=1,2,3} \alpha_{j} \delta_{s_{j}}
$$

has the barycenter $s$ and the support in $I$, there exists, by Choquet's theorem, an extremal measure $v$ on $I$ such that $\mu \prec v$. Suppose that $v=v_{B}$ with $B$ an abelian $W^{*}$-subalgebra of $\mathscr{R}_{s}^{\prime}$; by Cartier-Fell-Meyer theorem the measure $\mu$ must be a discrete $B$-measure, this implies that $b_{j} \in B, j=1,2,3$, this contradicts the hypothesis $b_{1} b_{2} \neq b_{2} b_{1}$. q.e.d. 


\section{The Greatest Lower Bound of Extremal Invariant Measures}

Lemma 2. Let $\left(B_{k}\right)_{k \in K}$ be a set of abelian $W^{*}$-subalgebra of $\pi_{s}(A)^{\prime}$ and $B=\bigcap_{k \in K} B_{k}$; $v_{B}^{s}$ is the greatest lower bound of the measure $v_{B_{k}}^{s}$ for the order $\prec$.

Proof. Let $v=v_{B}$ and $v_{k}=v_{B_{k}}$; it is clear that $v \prec v_{k}, \forall k \in K$. Conversely let $\mu$ be a finite discrete measure of $\Omega(s)$ such that $\mu \prec v_{k}, \forall k \in K$; then $\mu$ is a discrete $B_{k}$, $\forall k \in K$, hence $\mu$ is a discrete $B$-measure, therefore $\mu \prec v$. q.e.d.

We have seen that, in general, there are many extremal measures on $I$ associated with an invariant state $s$, but we have the following canonical measure (which is not an extremal measure unless $\mathscr{R}_{s}^{\prime}$ is abelian).

Proposition 2. Let $s$ be an invariant state, let $B_{s}=$ Center $\left(\mathscr{R}_{s}^{\prime}\right)$; if $A$ is separable then the $B_{s}$-measure $v_{B}^{s}$ of $s$ is the greatest lower bound of all extremal measures on I with barycenter s for Choquet-Bishop-de Leeuw order.

Proof. Let $v=v_{B_{s}}^{s}$; as the center of $\mathscr{R}_{s}^{\prime}$ is exactly the intersection of all maximal abelian $W^{*}$-algebras of $\mathscr{R}_{s}^{\prime}$, it is clear by the above Lemma 2 that

$$
v=\inf \left\{v_{B} / B \text { maximal abelian } W^{*} \text {-subalgebra of } \mathscr{R}_{s}^{\prime}\right\}
$$

It is sufficient to prove that $\nu \prec \mu$, for all extremal measures $\mu$ on $I$ with barycenter $s$. Since $v$ is a vague limit of discrete $B$-measures $v_{\left\{b_{j}\right\}}$, it is sufficient to show that for such a $v_{\left\{b_{j}\right\}}$, we have $v_{\left\{b_{j}\right\}} \prec \mu$. Let $\sum_{i=1}^{p} \beta_{i} \delta_{Q_{i}} \prec \mu, t_{i} \in \mathscr{R}_{s^{+}}^{\prime}$ such that (as in Lemma 0), if $\beta_{i}=\left\langle t_{i} \xi_{s}, \xi_{s}\right\rangle$, we have $\varrho_{i}(a)=\frac{1}{\beta_{i}}\left\langle\pi_{s}(a) t_{i} \xi_{s}, \xi_{s}\right\rangle, \forall a \in A$, and $\sum_{i=1}^{p} t_{i}=1$. Consider the discrete measure $\mu^{\prime}=\sum_{j=1, i=1}^{n} \gamma_{i j}^{p} \delta_{s_{i j}}$, where

We have

$$
\begin{aligned}
\gamma_{i j} & =\left\langle t_{i} b_{j} \xi_{s}, \xi_{s}\right\rangle \\
s_{i j}(a) & =\frac{1}{\gamma_{i j}}\left\langle\pi_{s}(a) t_{i} b_{j} \xi_{s}, \xi_{s}\right\rangle, \quad \forall a \in A .
\end{aligned}
$$

In a similar way, we obtain

$$
\begin{aligned}
\sum_{i=1}^{p} \gamma_{i j} \delta_{s_{i j}}(\hat{a}) & =\sum_{i=1}^{p} \gamma_{i j} \frac{1}{\gamma_{i j}}\left\langle\pi_{s}(a) t_{i} b_{j} \xi_{s}, \xi_{s}\right\rangle \\
& =\left\langle\pi_{s}(a)\left(\sum_{i=1}^{p} t_{i}\right) b_{j} \xi_{s}, \xi_{s}\right\rangle \\
& =\left\langle\pi_{s}(a) b_{j} \xi_{s}, \xi_{s}\right\rangle \\
& =\alpha_{j} s_{j}(a), \quad \forall a \in A
\end{aligned}
$$

$$
\sum_{j=1}^{n} \gamma_{i j} \delta_{s_{i j}}(\hat{a})=\beta_{i} \varrho_{i}(a), \quad \forall a \in A
$$

Hence

$$
\begin{gathered}
v_{\left\{b_{j}\right\}} \prec \sum_{i=1}^{p}, \sum_{j=1}^{n} \gamma_{i j} \delta_{s_{i j}} \\
\sum_{i=1}^{p} \beta_{i} \delta_{\varrho_{i}} \prec \sum_{i=1}^{p}, \sum_{j=1}^{n} \gamma_{i j} \delta_{s_{i j}},
\end{gathered}
$$


Choosing an increasing filter $\sum_{i=1}^{p} \beta_{i} \delta_{\varrho_{i}}$ converging vaguely to $\mu$, the associated increasing filter $\gamma_{i j} \delta_{s_{i j}}$ (with $v_{\left\{b_{j}\right\}}$ fixed) converges also vaguely to $\mu$ (for $\mu$ is maximal in $\left.\Omega^{I}(s)\right)$; therefore

$$
v_{\left\{b_{j}\right\}} \prec \sum_{i=1}^{p} \gamma_{i j} \delta_{s_{i j}} \prec \mu .
$$

Hence $v \prec \mu$. q.e.d.

\section{Decomposition of Covariant States}

Proposition 3. Let $A$ be a $C^{*}$-algebra with identity, $G$ a topological group, $\tau$ a representation of $G$ into $\operatorname{Aut}^{*}(A), s$ a $\tau$-covariant state on $A$ (cf. [8]), $Z_{0}$ the $W^{*}$ subalgebra of invariant elements of Center $\left(A^{\prime \prime}\right)$; the $Z_{0}$-measure of $s$ is "concentrated" on the set of $Z_{0}$-pure states - namely if $\Delta$ is a Baire set in $E$ with $\Delta \bigcap \mathscr{F}_{Z_{0}}$ $=\Phi\left(\mathscr{F}_{Z_{0}}\right.$ is the set of $Z_{0}$-pure states on $\left.E\right)$ then $v(\Delta)=0$. If $s$ is $\tau$-invariant then $v$ is "concentrated" on the set of $Z_{0}$-pure invariant states $I \bigcap \mathscr{F} Z_{0}$.

Proof. Consider $\mathfrak{H}, \pi, \xi, U_{g}$ associated with the covariant state $s$; let $B=\pi\left(Z_{0}\right)$, $D$ the $C^{*}$-algebra generated by $\pi(A), U_{G}$ and $\pi(A)^{\prime}$; we have

$$
D^{\prime}=\pi(A)^{\prime} \cap U_{G}^{\prime} \cap \pi(A)^{\prime \prime}=B
$$

since $\pi\left(Z_{0}\right)$ is the set of invariant elements of the center of $\pi(A)^{\prime \prime}$ (cf. [8], Lemma 3); let $\tilde{E}$ be the set of states on $D$ and consider the state $\tilde{s}: \tilde{s}(d)=\langle d \xi, \xi\rangle, d \in D$; the group $G$ acts on $D$ in canonical way: $d \rightarrow U_{g} d U_{g}^{-1}, d \in D, g \in G$.

Let $\tilde{v}$ be the $B$-measure of $\tilde{s}$ on $\tilde{E}, \tilde{v}$ is an extremal measure on $\tilde{E}$ (cf. Lemma 1 ), let $\gamma$ be the natural continuous mapping of $\tilde{E}$ into $E$ (as in the proof of Theorem 1), it is clear that $v=\gamma(\tilde{v})$ is the $Z_{0}$-measure of $s$ on $E$.

Let $\Delta$ be a Baire subset of $E$ such that $\Delta \cap \mathscr{F}_{Z_{0}}=\Phi$, we shall show that $\gamma^{-1}(\Delta)$ $\cap \mathscr{E}(E)=\Phi$. Suppose that $\tilde{\phi} \in \gamma^{-1}(\Delta) \cap \mathscr{E}(E)$, the representation $\tilde{\pi}_{\tilde{\phi}}$ (with the same notations as in the proof of Theorem 1) of $D$ is covariant and irreducible

$$
\tilde{\pi}_{\tilde{\phi}}(D)^{\prime}=\tilde{\pi}_{\tilde{\phi}}(\pi(A))^{\prime} \cap \tilde{\pi}_{\tilde{\phi}}\left(\pi(A)^{\prime}\right)^{\prime} \cap \tilde{\pi}_{\tilde{\phi}}\left(U_{G}\right)^{\prime}=\{\text { scalars }\} .
$$

Let $\phi=\gamma(\tilde{\phi}), \pi_{1}=\tilde{\pi}_{\phi} \pi, \tilde{U}=\tilde{\pi}_{\circ} U$; we have (cf. [8] Lemma 3 with a trivial modification):

$$
\pi_{1}\left(Z_{0}\right)=\pi_{1}(A)^{\prime \prime} \cap \pi_{1}(A)^{\prime} \cap\left(\tilde{U}_{G}\right)^{\prime}
$$

or

$$
\pi_{1}\left(Z_{0}\right)=\tilde{\pi}_{\phi}(\pi(A))^{\prime \prime} \cap \tilde{\pi}_{\phi}(\pi(A))^{\prime} \cap \tilde{\pi}_{\tilde{\phi}}\left(U_{G}\right)^{\prime}
$$

The relation

$$
\tilde{\pi}_{\tilde{\phi}}\left(\pi\left(A^{\prime}\right)\right) \subset \tilde{\pi}_{\tilde{\phi}}(\pi(A))^{\prime}
$$

implies

$$
\tilde{\pi}_{\tilde{\phi}}\left(\pi(A)^{\prime}\right)^{\prime} \supset \tilde{\pi}_{\tilde{\phi}}(\pi(A))^{\prime \prime} .
$$

This last relation and (1), (2) give

$$
\pi_{1}\left(Z_{0}\right)=\{\text { scalars }\} .
$$


The cyclic representation $\pi_{\phi}$ of $A$ is a subrepresentation of $\pi_{1}$, therefore $\pi_{\phi}\left(Z_{0}\right)$ $=\{$ scalars $\}$ i.e. $\phi$ is $Z_{0}$-pure (cf. [8]) which contradicts the hypothesis; hence $v(\Delta)=\tilde{v}\left(\gamma^{-1}(\Delta)\right)=0$. q.e.d.

Remark. This proposition is an improvement of some results of GuichardetKastler (cf. [8]); the use of $\Sigma^{*}$-algebras provides another method giving other properties of the $Z_{0}$-measures, particularly for successive decompositions (cf. [3]).

\section{Continuity of the Integral Representation}

Let $s$ be a fixed state on $A$, we shall study a certain form of continuity of the mapping: $B \rightarrow v_{B}^{s}$, with $B$ an abelian $W^{*}$-subalgebra of $\pi_{s}(A)^{\prime}$.

Proposition 4. Let $\left(B_{k}\right)_{k \in K}$ be an increasing filter of abelian $W^{*}$-subalgebras of $\pi_{s}(A)^{\prime}$, let $B$ be the abelian $W^{*}$-subalgebra generated by $\bigcup_{k \in K} B_{k}$. The set of $B_{k^{-}}$ measures $v_{k}$ of $s$ in an increasing filter (for the order $\prec$ ) converging vaguely to the $B$-measure $v$ of $s$.

Proof. We can identify $\overline{[B \xi]}=L^{2}(X, \mathscr{B}, \mu)$ and $B=L^{\infty}(X, \mathscr{B}, \mu)$, with $(X, \mathscr{B}, \mu)$ a probability space and $B_{k}=L^{\infty}\left(X, \mathscr{B}_{k}, \mu\right)$ where $\left(\mathscr{B}_{k}\right)_{k \in K}$ is an increasing filter of $\sigma$-subalgebras of $\mathscr{B}$.

The set $\left(v_{k}\right)_{k \in K}$ is an increasing filter of elements of $\Omega(s)$ bounded by $v$. For every continuous convex function $f$ on $E\left(v_{k}(f)\right)_{k \in K}$ is an increasing filter of real numbers bounded by $v(f)$, let $\mu(f)=\lim _{K} v_{k}(f)$, we have $\mu(f) \leqq v(f)$; since the set of functions $f-g$, with $f, g$ continuous convex, are dense in $C(E)$, there exists a measure $\mu \in \Omega(s)$ such that $v_{k} \vec{K} \mu$ for the vague topology, and $\mu \prec v$.

To prove that $\mu=v$, it is sufficient to show that $v \prec \mu$ (for $\prec$ is an order); using Cartier-Fell-Meyer theorem, it is sufficient to prove $v_{\left\{b_{j}\right\}} \prec \mu$ for all discrete $B$-measures $v_{\left\{b_{j}\right\}}$.

Let $b_{j}^{k}=E^{\mathscr{B}_{k}} b_{j}$, where $E^{\mathscr{B}_{k}}$ denotes the conditional expectation with respect

to $\mathscr{B}_{k}$; we have $b_{j}^{k} \in B_{k+}, \sum_{j} b_{j}^{k}=1$, let $v_{\left\{b_{j}^{k}\right\}}$ the associated discrete $B_{k}$-measure.
We have
$v_{\left(b_{j}\right)} \prec v_{k} \prec \mu$.

$$
v_{\left\{b_{j}^{k}\right\}} \prec v_{k} \prec \mu \text {. }
$$

As the order $\prec$ is vaguely closed, it is sufficient to prove that

$$
v_{\left\{b_{j}^{k}\right\}} \overrightarrow{\boldsymbol{K}} v_{\left\{b_{j}\right\}} \text { vaguely, } j=1, \ldots, n
$$

i.e.

or

$$
\delta_{s_{j}^{k}} \vec{K} \delta_{s_{J}} \text { vaguely, } j=1, \ldots, n
$$

$$
\begin{gathered}
s_{j \vec{K}}^{k} s_{j} \text { weakly, } \quad j=1, \ldots, n \\
s_{j}^{k}(a)_{\vec{K}} s_{j}(a), \quad \forall a \in A, \quad j=1, \ldots, n .
\end{gathered}
$$

As $E^{\mathscr{B}_{k}} b_{j} \vec{K} b_{j}$ for the $L^{1}$-norm topology, we have the convergence also for the $\sigma\left(L^{\infty}, L^{1}\right)$-topology, since the set $\left(E^{\mathscr{B}_{k}} b_{j}\right)_{k \in K}$ is uniformly bounded (cf. [11] IV.3.2.). Therefore

$$
s_{j}^{k}(a)=\left\langle\pi(a) b_{j}^{k} \xi, \xi\right\rangle \overrightarrow{\boldsymbol{K}}_{\vec{\lambda}} s_{j}(a)=\left\langle\pi(a) b_{j} \xi, \xi\right\rangle
$$

for all $a \in A$, and $j=1, \ldots, n$.

The proof is complete. 
Proposition 5. Let $\left(B_{k}\right)_{k \in K}$ be a decreasing filter of abelian $W^{*}$-subalgebras of $\pi_{s}(A)^{\prime}$ and let $B=\bigcap_{k \in K} B_{k}$. The set $\left(v_{k}\right)_{k \in K}$ of the $B_{k}$-measure of $s$ is a decreasing filter (for the order $\prec$ ) converging vaguely to the B-measure $v$ of $s$.

Proof. We show as in the proof of Proposition 4 that $v_{k}$ converges vaguely to its greatest lower bound, and by the Lemma 2, this greatest lower bound is precisely the $B$-measure $v$. q.e.d.

Proposition 6. Let $\left(B_{k}\right)_{k \in K}$ be a set of abelian $W^{*}$-subalgebras of $\pi_{s}(A)^{\prime}$, indexed by a filter $K$, such that $B_{k}$ commutes with $B_{k^{\prime}}$, for $k, k^{\prime} \in K$; let $\lim _{K} \sup B_{k}=\bigcap_{k \in k} \bigvee_{l \geqq k} B_{l}$ and $\lim _{K} \inf B_{k}=\bigvee_{k \in K} \bigcap_{l \geqq k} B_{l}$, where $\bigvee_{l \geqq k} B_{l}$ denotes the $W^{*}$-algebra generated by $\left(B_{l}\right)_{l \geqq k}$. If $\lim _{K} \sup B_{k}=\lim _{K} \inf B_{k}=B$, then the filter $\left(v_{B_{k}}^{s}\right)_{k \in K}$ converges vaguely to $v_{B}^{s}$.

Proof. Let

$$
C_{k}=\bigvee_{l \geqq k} B_{l} \quad \text { and } \quad D_{k}=\bigcap_{l \geqq k} B_{l} ; \quad\left(C_{k}\right)_{k \in K}
$$

(resp. $\left.\left(D_{k}\right)_{k \in K}\right)$ is a decreasing (resp. increasing) filter of abelian $W^{*}$-subalgebras of $\pi_{s}(A)^{\prime}$; the Proposition 4 and 5 show that $v_{C_{k} \vec{K}} v_{B}$ and $v_{D_{k}} \vec{R} v_{B}$ for the vague topology; as we have $D_{k} \subset B_{k} \subset C_{k} ; v_{D_{k}} \prec v_{B_{k}} \prec v_{C_{k}}$, this implies that for every continuous convex function $f$ on $E$

$$
v_{D_{k}}(f) \leqq v_{B_{k}}(f) \leqq v_{C_{k}}(f) .
$$

As $v_{D_{k}}(f) \rightarrow v_{B}(f)$ and $v_{C_{k}}(f)_{\vec{K}} v_{B}(f) ; v_{B_{k}}(f)_{\vec{K}} v_{B}(f)$. Since the functions of the form $f-g$, with $f, g$ continuous convex, are dense in $C(E) ; v_{B_{k}} \vec{K} v_{B}$ vaguely. q.e.d.

Definition. Let $\left(B_{k}\right)_{k \in K}$ be a set of abelian $W^{*}$-subalgebras of $\pi_{s}(A)^{\prime}$, such that $B_{k}$ commutes with $B_{k^{\prime}}$, for $k, k^{\prime} \in K$; we say that $\left(B_{k}\right)_{k \in K}$ is independent if

$$
\left\langle b_{k} b_{l} \ldots b_{m} \xi_{s}, \xi_{s}\right\rangle=\left\langle b_{k} \xi_{s}, \xi_{s}\right\rangle\left\langle b_{l} \xi_{s}, \xi_{s}\right\rangle \ldots\left\langle b_{m} \xi_{s}, \xi_{s}\right\rangle
$$

for all $b_{k} \in B_{k}, b_{l} \in B_{l}, \ldots, b_{m} \in B_{m}$, and $k, l, \ldots, m$ distinct in $K$ (cf. [11]).

Corollary 1. Let $\left(B_{k}\right)_{k \in K}$ be a set of independent abelian $W^{*}$-subalgebras of $\pi_{s}(A)^{\prime}$, indexed by a filter $K$. The filter $\left(v_{B_{k}}^{s}\right)_{k \in K}$ converges vaguely to the Dirac measure $\delta_{s}$ at the point $s$.

Proof. By the $\{0,1\}$-law (cf. [11]) $\lim _{K} \inf B_{k}=\lim _{K} \sup B_{k}=\{$ scalars $\}$ and so $v_{B}^{s}=\delta_{s}$. q.e.d.

\section{Characterization of Simplicial Systems - A Converse of Haag - Kastler - Michel Theorem}

Let $s$ be an invariant state of $A, A^{\prime \prime}$ be the $W^{*}$-envelope of $A, Z$ its center, $\mathscr{J}\left(\right.$ resp. $\left.\mathscr{J}_{s}, Z_{s}^{G}\right)$ the set of $G$-invariant elements of $A^{\prime \prime}\left(\right.$ resp. $\left.\pi_{s}\left(A^{\prime \prime}\right), \pi_{s}(2)\right) e\left(\right.$ resp. $\left.e_{s}\right)$ the finite part of the system $\left(A^{\prime \prime}, G\right)$ resp. $\left(\pi_{s}\left(A^{\prime \prime}\right) ; G\right), K_{s}$ the projection on $\mathfrak{S}^{G}$ the space of $\left(U_{G}\right)$-invariant vectors of $\mathfrak{G}, L_{s}$ the central support of $K_{s}$ in $\mathscr{J}_{s}$ (cf. [4]). 
We say that the invariant state $s$ satisfies the condition

$$
\begin{array}{lll}
\left(C_{1}\right) & \text { if } \quad \mathscr{J}_{s}=\mathbb{C} 1_{\mathfrak{H}} \\
\left(C_{2}\right) & \text { if } \quad\left(\mathscr{J}_{s}\right)_{e_{s}}=\mathbb{C}_{e_{s}} \\
\left(C_{3}\right) & \text { if } \mathfrak{H}^{G}=\mathbb{C} \xi_{s} \quad \text { (or } s \text { is weakly clustering (cf. [12])) } \\
\left(C_{4}\right) \text { if } \mathscr{R}_{s}^{\prime}=\mathbb{C} 1 \quad \text { (or } s \text { is ergodic (cf. [12])) } \\
\left(C_{5}\right) \text { if } \quad Z_{s}^{G}=\mathbb{C} 1 \quad \text { (or } s \text { is centrally ergodic (cf. [8])). }
\end{array}
$$

Let $\mathscr{E}_{i}$ be the set of invariant states satisfying $\left(C_{i}\right), i=1, \ldots, 5$; it is clear that $\mathscr{E}_{1} \subset \mathscr{E}_{2} \subset \mathscr{E}_{3} \subset \mathscr{E}_{4} \subset \mathscr{E}_{5}$

Throughout this paragraph, we assume $A$ separable, $G$ locally compact separable acting (norm-) continuously on $A$.

Theorem 2. The following conditions are equivalent:

(i) The system $(A ; G)$ is simplicial.

(ii) $\mathscr{E}_{3}=\mathscr{E}_{4}$ (i.e. $\{$ ergodic states $\}=\{$ weakly clustering states $\}$ ).

Proof. By [4] the condition (i) means that $A$ is $G$-abelian; the implication (i) $\Rightarrow$ (ii) is due to Ruelle (cf. $[12,13]$ ).

(ii) $\Rightarrow$ (i): let $s \in I, B$ a maximal abelian sub- $W^{*}$-algebra of $\mathscr{R}_{s}^{\prime}$, let

$$
\begin{aligned}
\mathfrak{H}_{s} & =\int_{X}^{\oplus} \mathfrak{G}_{x} d v(x) \\
\xi_{s} & =\int^{\oplus} \xi_{x} d v(x) \\
U_{g} & =\int_{\oplus}^{\oplus} U(x)_{g} d v(x), \quad g \in G . \\
\pi & =\int^{\oplus} \pi_{x} d v(x) .
\end{aligned}
$$

be a decomposition of $\mathfrak{G}_{s}, \xi_{s}, U_{g}, \pi$ associated to $B$ (cf. $\left.[5,6]\right)$ satisfying for all $x \in X$ :

$$
\begin{aligned}
\pi_{x}\left(\tau_{g} a\right) & =U(x)_{g} \pi_{x}(a) U(x)_{g}^{*}, \quad \forall a \in A, \quad \forall g \in G . \\
\left(\pi_{x}(A) \cup U(x)_{G}\right)^{\prime} & =C 1_{\mathfrak{H}} \quad(\text { cf. }[5] \text { p. 172) } \\
U(x)_{g} \xi_{x} & =\xi_{x}, \quad \forall g \in G \\
\mathfrak{H}_{x} & =\pi_{x}(A)^{\prime \prime} \xi_{x} .
\end{aligned}
$$

Let $s_{x}=\omega_{\xi_{x}}$, we identify $\mathfrak{G}_{x}=\mathfrak{G}_{s_{x}}, \pi_{x}=\pi_{s_{x}}$ : the state $s_{x}$ is ergodic (i.e. satisfying $C_{4}$ ) hence weakly clustering by the hypothesis i.e. $\left(\mathfrak{H}_{x}\right)^{G}=\mathbb{C} . \xi_{s_{x}}, \forall x \in X$. As $K_{s} \in\left(U_{G}\right)^{\prime}$ (cf. [13]), $K_{s}$ is decomposable: $K_{s}=\int_{x}^{\oplus} K(x) d v(x)$, and it is clear that $K(x) \mathfrak{H}_{x} \subset \mathfrak{H}_{x}^{G}$ $=\mathbb{C} \cdot \xi_{x}$.

Therefore

$$
\begin{aligned}
{\left[K_{s} \pi_{s}(a) K_{s}, K_{s} \pi_{s}(b) K_{s}\right] } & =\int_{x}^{\oplus}\left[K(x) \pi_{x}(a) K(x), K(x) \pi_{x}(b) K(x)\right] d v(x) \\
& =\int^{\oplus}\left[K(x) \pi_{x}(b) K(x), K(x) \pi_{x}(a) K(x)\right] d v(x) \\
& =\left[K_{s} \pi_{s}(b) K_{s}, K_{s} \pi_{s}(a) K_{s}\right]
\end{aligned}
$$


for all $a, b \in A$, and all $s \in I$; therefore $A$ is $G$-abelian (cf. [12],13]) i.e. $(A, G)$ is simplicial.

Theorem 3. The following conditions are equivalent:

(i) $(A, G)$ is quasi-large (i.e. $\mathscr{J}_{e}=\left(Z^{G}\right)_{e}$ cf. [4])

(ii) $\mathscr{E}_{4}=\mathscr{E}_{5}$ (i.e. $\{$ ergodic states $\}=\{$ centrally ergodic states $\left.\}\right)$

(iii) $\mathscr{E}_{3}=\mathscr{E}_{5}$

(iv) $\mathscr{E}_{2}=\mathscr{E}_{5}$.

Proof. The implication (i) $\Rightarrow$ (ii) is due to Nagel (cf. [10]) and is a generalization of a result of Haag, et al. (cf. [14] Theorem 3.5.10 p. 150); it is clear that (i) $\Rightarrow$ (iii) $\Rightarrow$ (ii).

(ii) $\Rightarrow(\mathrm{i})$ : Let $s \in I, \mu_{s}^{G}$ be its $\pi_{s}\left(Z^{G}\right)$-measure, $\mu_{s}^{G}$ is supported by $I$ (Lemma 0 ) and the set of centrally ergodic states (or $Z^{G}$-pure states by Proposition 3 ) therefore, the hypothesis implies that $\mu_{s}^{G}$ is supported by $\mathscr{E}(I)=\mathscr{E}_{4}$, hence by Theorem 1 , $\pi_{s}\left(Z^{G}\right)$ is maximal abelian in $\mathscr{R}_{s}^{\prime}$; as $\pi_{s}\left(Z^{G}\right) \subset$ Center $\left(\mathscr{R}_{s}^{\prime}\right)$, it follows that $\mathscr{R}_{s}^{\prime}=\pi_{s}\left(Z^{G}\right)$, $\forall s \in I$; therefore $(A ; G)$ is quasi-large (cf. [4] Theorem 2 ).

Theorem 4. The following conditions are equivalent:

(i) The system $(A ; G)$ is large (cf. [7] or equivalently $\mathscr{J}_{e} \subset Z^{G} c f$. [4]).

(ii) For all $s \in I$, we have $L_{s}=1_{\mathfrak{H}_{s}}$ and $\pi_{s}\left(Z^{G}\right)=\pi_{s}(\mathscr{J})$.

(iii) $\mathscr{E}_{1}=\mathscr{E}_{5}$.

Proof. (i) $\Rightarrow$ (iii). Let $s \in \mathscr{E}_{5}$, the system $\left(\pi_{s}(A)^{\prime \prime} ; G\right)$ is finite and $\mathscr{J}_{s}=Z_{s}^{G}=\mathbb{C}_{1} 1_{\mathfrak{H}_{s}}$; therefore $\mathscr{E}_{1}=\mathscr{E}_{5}$.

(iii) $\Rightarrow$ (ii) By the Theorem 3 , the system $(A ; G)$ is quasi-large; let $s \in I$, we have $\left(Z_{s}^{G}\right)^{\prime} \supset \mathscr{J}_{s} \supset Z_{s}^{G}$; as in the proof of Theorem 2 , let $\mathfrak{H}_{s}=\int_{x}^{\oplus} \mathfrak{H}_{x} d v(x) \ldots$ be a decomposition associated to $Z_{s}^{G}$, let

$$
\begin{aligned}
K_{s} & =\int_{x}^{\oplus} K(x) d v(x) \\
L_{s} & =\int_{x}^{\oplus} L(x) d v(x)
\end{aligned}
$$

as $\mathscr{J}_{s}$ is decomposable and $L_{s} \in \operatorname{Center}\left(\mathscr{J}_{s}\right)$, it follows that $L(x) \in \operatorname{Center}\left(Z_{s}(x)\right)$ for almost all $x \in X$. Hence by the hypothesis $L(x)=1_{\mathfrak{H}_{x}}$ a.e.; therefore

$$
L_{s}=\int_{x}^{\oplus} 1_{\mathfrak{H}_{x}} d v(x)=1_{\mathfrak{H}_{s}}, \quad \forall s \in I
$$

We have proved (ii).

The implication (ii) $\Rightarrow$ (i) is clear (cf. [4]).

\section{References}

1. Bishop, E., De Leeuw, K.: Ann. Inst. Fourier 9, 305-331 (1959)

2. Cartier,P., Fell,J.M.G., Meyer,P.A.: Bull. Soc. Math. France 92, 435-445 (1964)

3. Dang-Ngoc-Nghiem: $\Sigma^{*}$-Algèbres, Probabilités non-commutatives et Applications. Mémoires de la Sté. Mathématique de France. $n^{0}$ 35, 145-189 (1973)

4. Dang-Ngoc-Nghiem, Ledrappier, F.: Sur les systèmes dynamiques simpliciaux. C. R. Acad. Sc. Paris t. 277, Série A, pp. $777-779$ (1973) 
5. Dixmier, J.: Les algèbres d'opérateurs dans l'espace hilbertian. Paris: Gauthier-Villars 1969

6. Dixmier, J.: Les $C^{*}$-algèbres et leurs représentations. Paris: Gauthier-Villars 1969

7. Doplicher,S., Kastler,D., Størmer, E.: J. Funct. Anal. 3, 419-434 (1969)

8. Guichardet,A., Kastler, D.: J. Math. Pures Appl. 49, 349-380 (1970)

9. Meyer, P.A.: Probabilités et potentiel. Paris: Hermann 1966

10. Nagel, B.: Commun. math. Phys. 26, 247-258 (1972)

11. Neveu, J.: Bases Mathématiques de Calcul des Probabilités. Paris: Masson et Cie 1970

12. Ruelle, D.: Statistical Mechanics. New York: Benjamin 1969

13. Ruelle, D.: J. Funct. Anal. 6, 116-151 (1970)

14. Sakai, S.: $C^{*}$-algebras and $W^{*}$-algebras. Berlin-Heidelberg-New York: Springer 1971

Communicated by H. Araki

\author{
Dang-Ngoc-Nghiem \\ Laboratoire de Probabilités \\ Université Paris VI \\ Tour 56 \\ 9, quai Saint Bernard \\ F-Paris 5è, France
}


